\title{
Tuning the conductivity along atomic chains by selective chemisorption
}

\author{
F. Edler, ${ }^{1}$ I. Miccoli, ${ }^{1}$ J. P. Stöckmann, ${ }^{1}$ H. Pfnür, ${ }^{1,2}$ C. Braun, ${ }^{3}$ S. Neufeld, ${ }^{3}$ S. Sanna, ${ }^{3}$ W. G. Schmidt,${ }^{3}$ and C. Tegenkamp ${ }^{1,2, *}$ \\ ${ }^{1}$ Institut für Festkörperphysik, Leibniz Universität Hannover, Appelstraße 2, 30167 Hannover, Germany \\ ${ }^{2}$ Laboratorium für Nano- und Quantenengineering (LNQE), Leibniz Universität Hannover, Schneiderberg 39, 30167 Hannover, Germany \\ ${ }^{3}$ Lehrstuhl für Theoretische Materialphysik, Universität Paderborn, 33095 Paderborn, Germany \\ (Received 5 December 2016; revised manuscript received 2 February 2017; published 7 March 2017)
}

\begin{abstract}
Adsorption of $\mathrm{Au}$ on vicinal $\mathrm{Si}(111)$ surfaces results in growth of long-range ordered metallic quantum wires. In this paper, we utilized site-specific and selective adsorption of oxygen to modify chemically the transport via different channels in the systems $\mathrm{Si}(553)-\mathrm{Au}$ and $\mathrm{Si}(557)$-Au. They were analyzed by electron diffraction and four-tip STM-based transport experiments. Modeling of the adsorption process by density functional theory shows that the adatoms and rest atoms on $\mathrm{Si}(557)$-Au provide energetically favored adsorption sites, which predominantly alter the transport along the wire direction. Since this structural motif is missing on $\mathrm{Si}(553)-\mathrm{Au}$, the transport channels remain almost unaffected by oxidation.
\end{abstract}

DOI: 10.1103/PhysRevB.95.125409

\section{INTRODUCTION}

Atomic wires on surfaces represent prototype quasi-onedimensional (quasi-1D) systems for studying fundamental properties such as Peierls instabilities, formation of charge density waves, or dimensional crossover from Fermi to Luttinger liquid behavior [1]. Among others, Au-induced arrays of wires on vicinal $\mathrm{Si}(111)$ substrates $[\mathrm{Si}(h h k)$ in the following] attracted considerable interest recently since the strong spin-orbit coupling gives rise to spin-polarized surface bands. In case of $\mathrm{Si}(553)-\mathrm{Au}$, even spin-ordering phenomena are reported yielding a spin-liquid behavior [2,3].

Defects play a crucial role, particularly in such arrays of atomic wires, and many of the phenomena mentioned above are strongly affected by the presence of point defects. Several of these systems suffer from missing atoms within the 1D structures, often in consequence of a kinetic limitation during the self-assembled growth process. For instance, point defects in $\mathrm{Au}$ chains grown on various $\mathrm{Si}(h h k)$ surfaces reduce the conductivities along the wires drastically, while they can increase at the same time the hopping probabilities across the wires, thus leading to a much lower anisotropy as expected from corresponding photoemission data [4-6]. Conversely, vacancies can also have a stabilizing effect in these quasi-1D systems. As an example, the threefold periodicity in $\mathrm{Si}(553)-\mathrm{Au}$, which stems from charge ordering along the Si-honeycomb chain structure, appears at temperatures below $100 \mathrm{~K}$ in the clean system, but can be seen up to room temperature in the vicinity of defects $[7,8]$.

Adsorbates may change locally the surface potential, thus acting as defects [9]. Therefore, depending on details of the coupling strengths and adsorption sites, adsorbates can be used to tune specific properties [10,11]. In case of $\mathrm{Si}(111)-\mathrm{In}, \mathrm{e.g}$., the critical temperature $T_{C}$ for the metal insulator transition can be shifted to lower temperatures by adsorption of various atoms, which commonly destabilize the low-temperature phase. On the contrary, molecular oxygen atoms enhance cooperatively the interwire and intrawire coupling and lead to a stabilization of the insulating $(8 \times 2)$ configuration, which

\footnotetext{
*tegenkamp@fkp.uni-hannover.de
}

increases $T_{C}$ [12-15]. Moreover, on the $\mathrm{Si}(111)$-Au $(5 \times 2)$ $\mathrm{Si}$ adatoms are intrinsically present and even mandatory for the formation of the geometry with the lowest (free) energy $[16,17]$. The control of their density during preparation allows even tuning of the 1D band structure [18-20].

Compared to other systems, systematic investigations of the influence of adsorbates on $\mathrm{Si}(h h k)$-Au surfaces are still missing to date, possibly because of their complex structure and, consequently, their manifold adsorption behavior [21-23]. The Si(553)-Au surface consists of a double row of $\mathrm{Au}$ atoms, which substitute $\mathrm{Si}$ surface atoms and form a honeycomblike chain of $\mathrm{Si}$ reconstruction at the step edge (Si-HC). Si(557)-Au has a very similar structure except that the Au chain is formed by a single row of atoms. Most importantly, the Si(557)-Au surface hosts a further Si-adatom chain surrounded by rest atoms. As a result, the metallic surface states probed by surface transport are of different origin.

In a recent STM study, the adsorption of water on $\mathrm{Si}(553)$ $\mathrm{Au}$ and $\mathrm{Si}(557)$-Au was investigated. Various kinds of defects were identified as a result of the reaction process [22]. Thereby, the Si-HC was found to be a favored location of adsorption. Hydroxyl adsorption there is accompanied by a substantial gap opening of around $0.5 \mathrm{eV}$. Moreover, the Si adatoms turn out to be more reactive than the $\mathrm{Si}-\mathrm{HC}$ sites. However, they do not induce formation of an electronic gap. These findings led to the conclusion that the $\mathrm{Si}-\mathrm{HC}$ sites are relevant for electronic transport. Indeed, in an earlier transport study it was concluded that the adsorption-induced distortion of the Si-HC lattice is the main reason for the poor conductivity found in these systems [4].

In this paper, we investigated the adsorption of oxygen on $\mathrm{Si}(553)-\mathrm{Au}$ and $\mathrm{Si}(557)-\mathrm{Au}$ by means of electrical transport measurements, low-energy electron diffraction (LEED), and density functional theory (DFT). The change of conductivity for $\mathrm{Si}(557)-\mathrm{Au}$ along the wires is up to four times stronger compared to $\mathrm{Si}(553)$-Au. In agreement with DFT and LEED, molecular oxygen preferentially adsorbs at the Si-adatom sites which are present only on $\mathrm{Si}(557)-\mathrm{Au}$. On the latter surface, the oxidation of these $\mathrm{Si}$ atoms flattens the surface band originating from the Si rest atoms and reduces the conductivity. After saturation of these sites, further oxidation takes place at the $\mathrm{Si}-\mathrm{HC}$ edge and the opening of gap by rehybridization 
is confirmed by DFT. However, the sticking probability for $\mathrm{O}_{2}$ at the Si-HC sites is significantly lower and the reaction kinetics found in this regime are comparable to those seen on $\mathrm{Si}(553)-\mathrm{Au}$.

\section{EXPERIMENT AND COMPUTATIONAL METHODS}

All experiments were performed with low-doped (with phosphorus) Si crystals cut in (557) and (553) directions and with a bulk resistivity of $\rho=1700 \Omega \mathrm{cm}$ at room temperature. Samples of $1 \times 0.5 \mathrm{~cm}^{2}$ size were cut from Si wafers, cleaned with acetone and isopropyl alcohol in an ultrasonic bath, rinsed in pure water, and finally dried in a pure $\mathrm{N}_{2}$ flow. After this ex situ cleaning procedure, the samples were loaded into the ultrahigh-vacuum (UHV) chamber operating at a base pressure of $1 \times 10^{-10}$ mbar, degassed at $700{ }^{\circ} \mathrm{C}$ for several hours by direct-current (dc) heating, and finally flash annealed at $1150^{\circ} \mathrm{C}$ for a few seconds while a pressure below $1 \times 10^{-9}$ mbar was maintained. Au atomic chains were prepared on $\operatorname{Si}(553)$ and $\operatorname{Si}(557)$ surfaces by evaporation of 0.48 and $0.20 \mathrm{ML}$ at $650{ }^{\circ} \mathrm{C}$, respectively, using a flux-controlled $e$-beam heated evaporator. After Au deposition, the dc heating was turned off and samples were immediately cooled down to room temperature. Details of the structures were analyzed by high-resolution low-energy electron diffraction (SPA-LEED, transfer width is around $200 \mathrm{~nm}$ ). In addition, four-point-probe (4PP) transport experiments were performed in the same ultrahigh vacuum system using a four-tip scanning tunneling microscope (STM) equipped with a scanning electron microscope (SEM) for precise positioning of the STM tips. The tips were made via $\mathrm{NaOH}$ etching from pure $\mathrm{W}$ wires (diameter $0.25 \mathrm{~mm}$ ) with typical tip radii between 20 and $50 \mathrm{~nm}$ [24]. Oxygen dosage experiments were performed at a background pressure of $5 \times 10^{-9}$ mbar. All experiments were carried out at $300 \mathrm{~K}$ and performed while oxygen was dosed. The cross section for electron-beam-induced dissociation by the electron beams of LEED and SEM turned out to be so low that no effect was detected, as explicitly tested by adsorption experiments performed with blanked beams.

Density functional theory (DFT) calculations were performed within the generalized gradient approximation (GGA) using the PBE functional [25]. The electron-ion interaction was modeled within the projector augmented wave (PAW) method [26] as implemented in the Vienna ab initio simulation package (VASP) [27]. An energy cutoff of $410 \mathrm{eV}$ and a $k$-point mesh of $2 \times 9 \times 1$ were utilized to determine the structurally relaxed ground-state configurations. The $\mathrm{Si}$ surfaces were modeled within periodic supercells, which contain a material slab of six essentially bulklike Si bilayers plus a surface layer containing $\mathrm{Si}$ and $\mathrm{Au}$ surface atoms as well as adsorbed $\mathrm{O}$ species. The opposite side of the slab was terminated with atomic hydrogen. The lowest two bilayers were fixed in ideal bulk positions during the structural relaxation, while all other atoms were allowed to relax. The adsorption energies were calculated by positioning $\mathrm{O}_{2}$ molecules in various starting configurations on specific lateral surface sites of the relaxed $\mathrm{Si}(h h k)$-Au surface. Thereby, the system was relaxed under the constraint that the lateral position of one oxygen atom remained fixed. Subsequent calculations for the energetically most stable adsorption sites on the potential energy surfaces without any structural constraints were used to determine the most favorable $\mathrm{O}$ adsorption geometries. These calculations provided the input for band structure calculations that allow for an interpretation of the experimental data.

\section{RESULTS AND DISCUSSION}

Before presenting the transport results, we will start in the first section with the presentation of the atomic structures and their changes upon adsorption of oxygen (Sec. A). Thereafter, the transport properties of clean structures and the effect of oxygen adsorption are presented (Secs. B and C). By means of DFT, the adsorption sites and geometries for dissociative adsorption of molecular oxygen (from the gas phase) are determined and their impact on the band structure is discussed (Sec. D). Based on these findings, we finally derive an adsorption scenario, which quantitatively explains our transport data (Sec. E).

\section{A. Structure of the pristine surfaces and the effect of oxygen adsorption}

The successful growth of long-range-ordered Au-induced chain structures on $\mathrm{Si}(553)$ and $\mathrm{Si}(557)$ surfaces is nicely demonstrated by the LEED patterns shown in Figs. 1(a) and $1(\mathrm{~d})$, respectively. The patterns taken at $300 \mathrm{~K}$ reflect the atomistic details of the crystallographic structures shown in Figs. 1(c) and 1(f), which were derived from recent $a b$ initio total-energy calculations [19,28].

For $\mathrm{Si}(553)-\mathrm{Au}$, the step-step distance along the [11 $\overline{2}]$ direction is $d_{[11 \overline{2}]}^{553}=4 \frac{1}{3} a_{[11 \overline{2}]} / \cos \phi=14.7 \AA$, where $a_{[11 \overline{2}]}=3.32 \AA$ and $\phi=12.5^{\circ}$ is the miscut angle with respect to the (111) orientation. In perfect agreement with the experiment, this correlated step-step structure gives rise to a spot splitting in reciprocal space along the [11 $\overline{2}]$ direction, which amounts to $2 \pi / d=0.42 \AA^{-1}$ or, equivalently, $22.2 \%$ SBZ (surface Brillouin zone), if normalized to the reciprocal lattice vector of the $\mathrm{Si}(111)$ surface. Similarly, the $\operatorname{Si}(557)$ surface yields a step-step distance of $d_{[\overline{1} 12]}^{557}=$ $a_{[11 \overline{2}]} /\left(5 \frac{2}{3} a_{[11 \overline{2}]} / \cos 9.5^{\circ}\right)=21.9 \AA$, which refers to a spot splitting of $17.4 \%$ SBZ, consistent with previous studies [29-32].

Aside from these characteristic spots of the step train, the LEED patterns exhibit intensity streaks along the [11ㄹ] direction. Most importantly, although the LEED images of $\mathrm{Si}(557)-\mathrm{Au}$ and $\mathrm{Si}$ (553)-Au look similar, the origin for these streaks is very different. The $\times 2$ streaks for the (553) $\mathrm{Si}$-Au are ascribed to the double periodicity within the gold chains along the [110]direction [23,31], whereas the $\times 2$ streaks seen for $\mathrm{Si}(557)-\mathrm{Au}$ surface originate from the $\mathrm{Si}$ adatoms [2,33,34]. For $\mathrm{Si}(553)-\mathrm{Au}$, superstructure formation along and between the wires sets in upon cooling $[3,35]$, which is not of further interest for our experiments performed at $300 \mathrm{~K}$. Nonetheless, its absence at $300 \mathrm{~K}$ is indicative for a low concentration of missing Au atoms [7].

Despite these structural similarities, the surfaces behave differently upon adsorption of oxygen. Figures 1(b) and 1(e) show the LEED patterns after adsorption of $20 \mathrm{~L}$. For both substrates, the periodicity of the step train is unchanged upon 

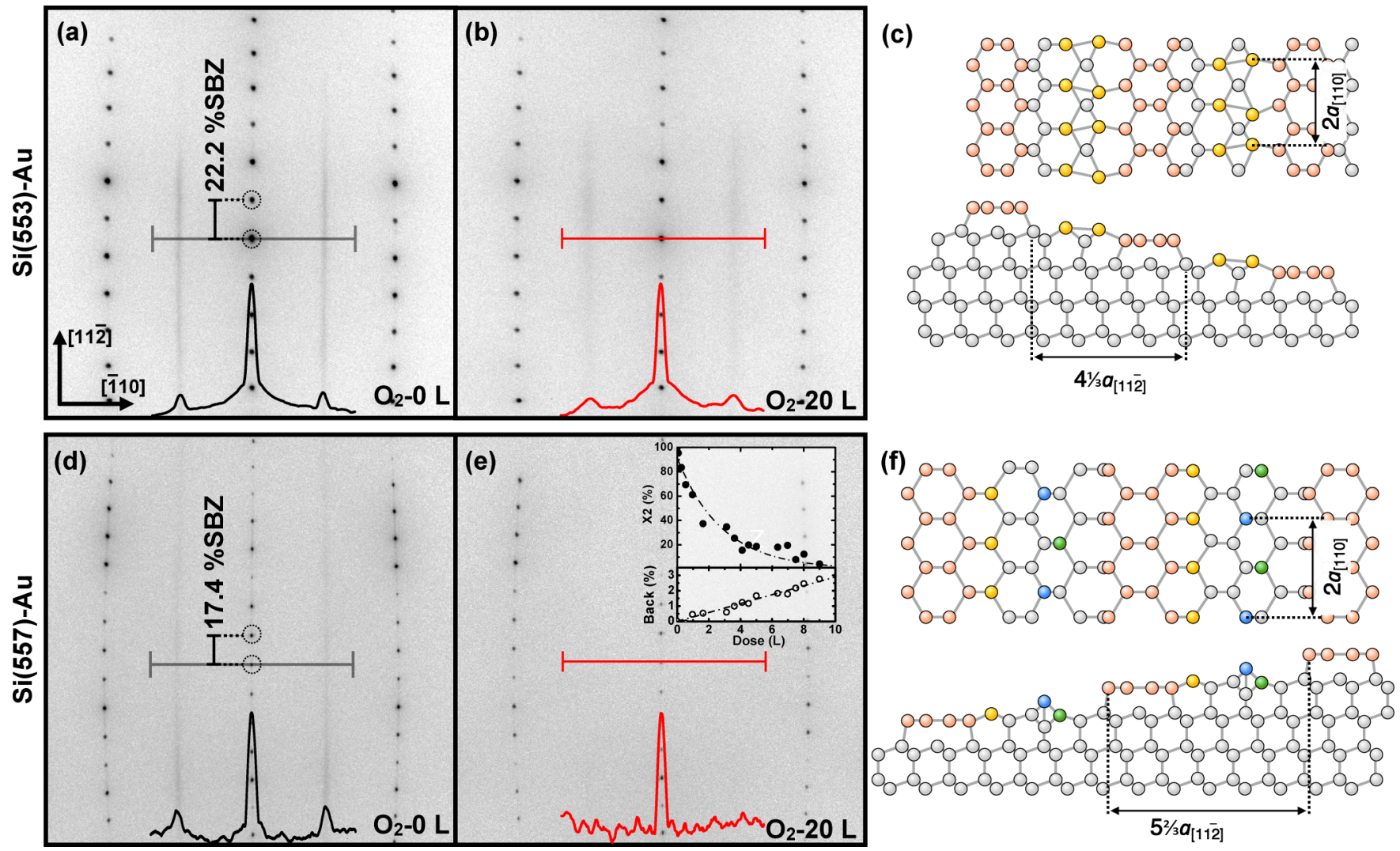

FIG. 1. SPA-LEED patterns of Si(553)-Au (a), (b) and Si(557)-Au surfaces (d), (e) before (a), (d) and after (b), (e) exposure to $20 \mathrm{~L}$ of molecular oxygen (electron energy $138 \mathrm{eV}$ ). Horizontal lines indicate where the line scans, shown at the bottom, were taken. The oxygen-induced gradual decrease of the $\times 2$ peak intensity and the linearly increasing background (back) are shown as a function of oxygen dose in the inset of (e). (c), (f) Show the corresponding crystal structures for the $\mathrm{Si}(553)-\mathrm{Au}$ and $\mathrm{Si}(557)$-Au, respectively [19,28]. The Au atoms, $\mathrm{Si}$ adatoms, rest atoms, and Si-honeycomb chain atoms are colored in yellow, blue, green, and orange, respectively.

adsorption. Remarkably, however, the $\times 2$ streaks induced by the dimerization of the Au chains on $\mathrm{Si}(553)$-Au are very little affected by oxygen adsorption, while these streaks in case of $\mathrm{Si}(557)$-Au have completely vanished, as demonstrated by the inset of Fig. 1(e), which shows the normalized peak intensity of the $\times 2$ spot as a function of the oxygen dose. Already after a dosage of $5 \mathrm{~L}$ the long-range order along the $\mathrm{Si}$ adatoms is entirely destroyed. Assuming that the ordering for LEED is effectively destroyed if every second Si dimer is occupied (on average), we can infer that the sticking coefficient is in the order of $10 \%-20 \%$. Within the data acquisition time for LEED, the dose is around $0.2 \mathrm{~L}$, i.e., five events of chemisorption take place within the transfer width of our LEED system. The linear increase of the background, also shown in the inset, is indicative for a random adsorption. The fact that the background increases even further shows that other sites become occupied by oxygen as well. DFT calculations confirmed that the dissociative adsorption of molecular oxygen takes place selectively at the $\mathrm{Si}$-adatom and rest-atom sites (see below). We will show that this has direct consequences for the electronic transport.

\section{B. Transport properties of the clean surfaces}

In order to perform surface-sensitive transport measurements, the W tips of our multiprobe STM system were sequentially approached in a feedback-controlled manner onto the sample surface, thus avoiding severe damages to the probes and to the atomic chains.

As exemplarily shown in Fig. 2(a) for $\mathrm{Si}(553)-\mathrm{Au}$, a square configuration was adopted for all measurements shown here since this geometry allows to measure the parallel and perpendicular components of the conductance independently. For each orientation of this configuration with respect to the crystallographic directions of the sample, IV measurements were performed by ramping the current between two probes (parallel or perpendicular to the chains) from -1 and $1 \mu \mathrm{A}$ while measuring the corresponding voltages between the other contacts. The different slopes of the IV curves, i.e., different resistances $R$, shown in Fig. 2(b) reflect already the anisotropy of the conductivity components of the surface. Since the orientation of the atomic wires on the surface is known, the conductivities $\sigma_{\|}$and $\sigma_{\perp}$ of the atomic wires can be easily deduced by $R_{\|(\perp)}=\left(2 \pi \sqrt{\sigma_{\|} \sigma_{\perp}}\right)^{-1} \ln \left(1+\sigma_{\|(\perp)} / \sigma_{\perp(\|)}\right)$ $[36,37]$. Moreover, by gradually varying the orientation angle $\theta$ of the four-tip setup with respect to the crystallographic directions of the surfaces, the modulation of the $R(\theta)$ curve in Fig. 2(c) allows to confirm independently the orientation of the atomic wires. For further details on the $R(\theta)$ function, the reader is referred to Refs. [36,37].

Applying the rotational square method to the $\mathrm{Si}(553)-\mathrm{Au}$ system, the best-fit yields conductivity values of $\sigma_{\|}=239 \mu \mathrm{S}$ and $\sigma_{\perp}=186 \mu \mathrm{S}$ leading to a ratio of $\sigma_{\|} / \sigma_{\perp} \approx 1.3$ at $300 \mathrm{~K}$. 

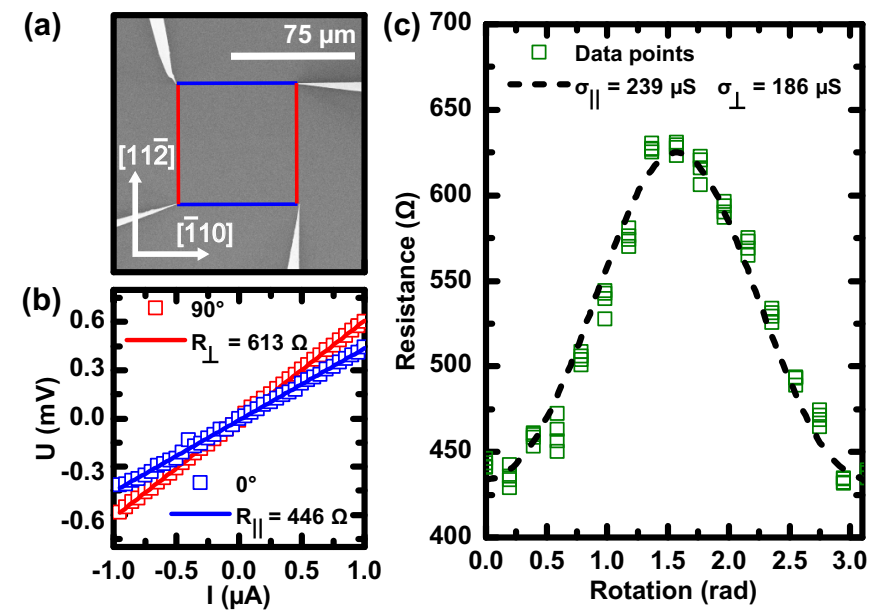

FIG. 2. (a) SEM image of the four STM tips used for transport measurements. A characteristic square geometry is shown exemplarily for $\mathrm{Si}(553)-\mathrm{Au}$. (b) Current-voltage (IV) curves obtained for two distinct configurations [current along the red (perpendicular direction) and blue lines (parallel direction), respectively]. From the slopes the resistances are determined and plotted as a function of the orientation of the squared arrangement w.r.t. the crystallographic directions of the surface. (c) Resistances multiply measured using the rotational square method ( $\pi / 2$ refers to current along the [112] direction, i.e., across the steps). The dashed line is a fit to the experimental data and allows to derive the conductivities along $\left(\sigma_{\|}\right)$and across $\left(\sigma_{\perp}\right)$ the array of wires [36].

This value is slightly smaller than the ones typically measured for the Si(111)-4 × 1-In system [38,39], but in close agreement with previous investigations of the $\mathrm{Au}-\mathrm{Si}(h h k)$ system [4,5]. We performed a similar measurement and analysis for the $\mathrm{Si}(557)-\mathrm{Au}$ system. For this system we obtained values of $\sigma_{\|} \approx 220 \mu \mathrm{S}$ and $\sigma_{\perp} \approx 160 \mu \mathrm{S}$ yielding a similar anisotropy.

We want to mention that the absolute values slightly vary from sample to sample due to different defect concentrations during preparation. Nonetheless, for all systems we measured at $300 \mathrm{~K}$ anisotropies ranging between 1.3 and 1.7. We showed recently for the $\mathrm{In} / \mathrm{Si}(111)$ system that line defects along the surface, e.g., substrate steps, can apparently increase the conductance anisotropy [38]. Therefore, the low values measured here are in principle indicative for atomically flat ensembles of wires. On the other hand, the ARPES data reveal quasi-1D Fermi surfaces. Hence, large anisotropy values are expected if only surface bands provide the transport channels [19]. Therefore, the finite resistance measured along the [112] direction must be due parasitic bulk channels, i.e., defect-induced modifications of the Si bulk bands, e.g., band bending $\left(\sigma_{\mathrm{SCL}}\right)[39,40]$, which reduce the expected anisotropy from the surface states. Generally, surface defects should reduce $\sigma_{\|}$in a quasi-1D conductor, but may increase $\sigma_{\perp}$ due to more isotropic scattering, leading also to low anisotropy values measured for both $\mathrm{Si}(553)-\mathrm{Au}$ and $\mathrm{Si}(557)-\mathrm{Au}$. However, we will show below that the surface transport across the channels is almost unaffected upon oxygen adsorption, thus this transport channel will be neglected.
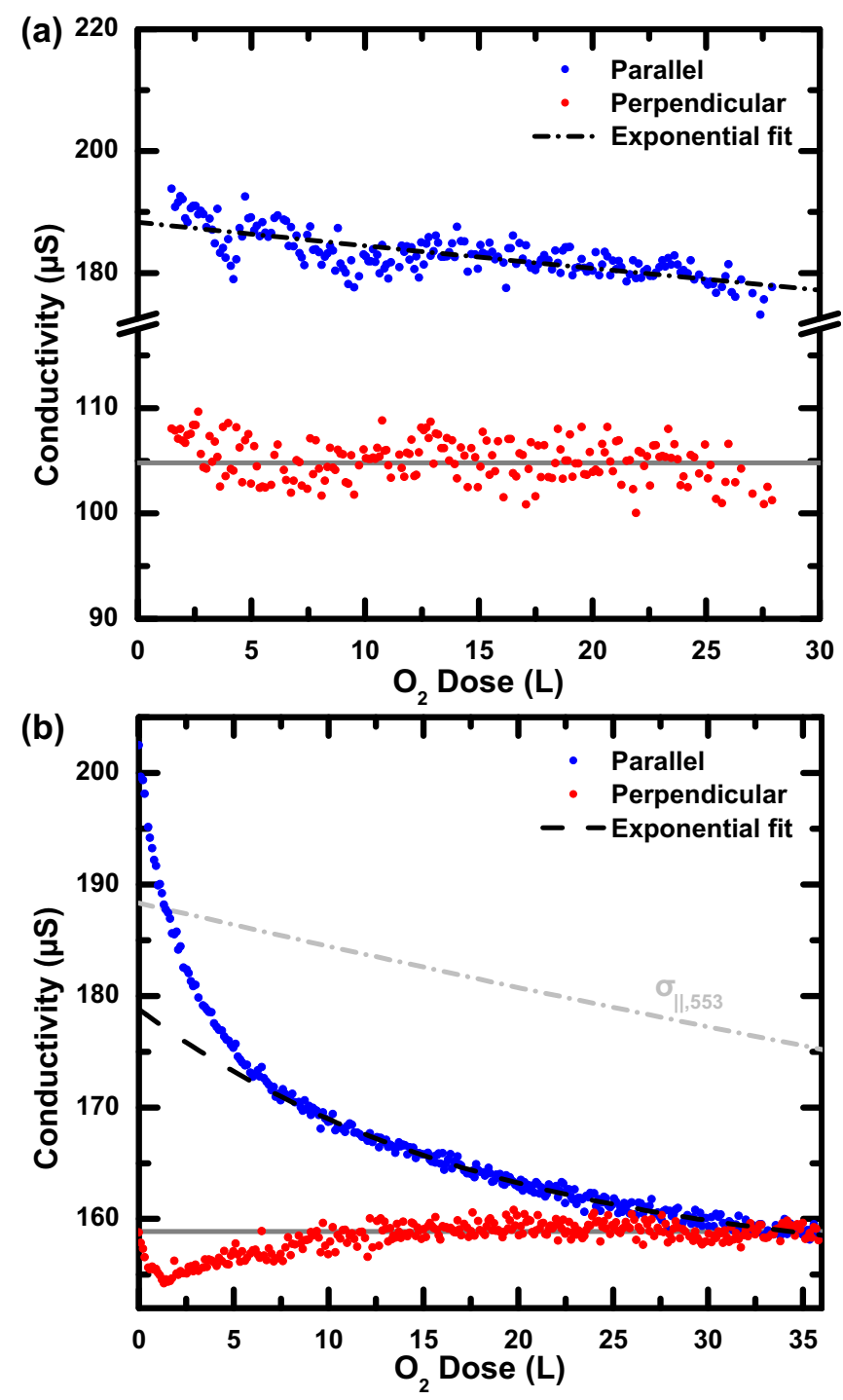

FIG. 3. Conductivity along $\left(\sigma_{\|}\right.$, blue $)$and perpendicular $\left(\sigma_{\perp}\right.$, red $)$ to the wire directions as a function of the $\mathrm{O}_{2}$ dose $D$ for $\mathrm{Si}(553)-\mathrm{Au}$ (a) and $\mathrm{Si}(557)-\mathrm{Au}$ (b). The dashed and dashed-dotted curves are exponential fits to the conductance on the $\mathrm{Si}(557)$ - $\mathrm{Au}$ and $\mathrm{Si}(553)-\mathrm{Au}$ surfaces, respectively. For further details, see text.

\section{Effect of oxygen adsorption on transport}

Figure 3 shows two conductivity components $\left(\sigma_{\|}, \sigma_{\perp}\right)$ as a function of the oxygen dose (in units of Langmuir (L) $=1.33 \times 10^{-6}$ mbar $\mathrm{s}$ ) for both $\mathrm{Si}(553)-\mathrm{Au}$ (a) and $\mathrm{Si}(557)-\mathrm{Au}(\mathrm{b})$. Apparently, the conductivity along the atomic chains is affected by $\mathrm{O}_{2}$ adsorption more strongly than $\sigma_{\perp}$. Moreover, while $\sigma_{\|}$for $\mathrm{Si}(553)$-Au is reduced only by $5 \%$ after $30 \mathrm{~L}$, leaving the sample anisotropy almost intact, the parallel conductivity of $\mathrm{Si}(557)-\mathrm{Au}$ decreases by $20 \%$ after the same exposure, and makes the initial transport anisotropy disappear.

Before we analyze in more detail the decay mechanisms along the wires, we will briefly discuss $\sigma_{\perp}$. This part of the conductivity for the $\mathrm{Si}(553)-\mathrm{Au}$ system remains constant [Fig. 3(a)], at least within the scatter of the data. In contrast, the corresponding component for $\mathrm{Si}(557)$-Au reveals a small conductivity drop of around $3 \%$ of the overall background 
signal until an $\mathrm{O}_{2}$ dose of $2.5 \mathrm{~L}$ is reached, before the conductivity slowly recovers towards its original value. We ascribe this tiny but measurable effect to a modification of band bending upon oxidation and consequently of $\sigma_{\mathrm{SCL}}$, which is strongest upon adsorption of roughly half of the reactive adsorption sites. For higher coverages, the effect starts to be compensated due to competing adsorbate-adsorbate interactions. Within this simple model, the $2.5 \mathrm{~L}$ would nicely corroborate the saturation dose of around $5 \mathrm{~L}$ seen in LEED for the annihilation of the $\times 2$ reconstruction on $\mathrm{Si}(557)$ $\mathrm{Au}$. The space charge layer (SCL) model was successfully applied for $\mathrm{Si}(111)-$ In [40]. Recently, systematic investigations done by Uetake et al. [39] showed that high-temperature treatments (above $1000{ }^{\circ} \mathrm{C}$ ) induce surface-near defects, which alter the initial doping profile. A reasonable scenario is the formation of interstitial $\mathrm{C}$ defects with typical concentrations of $10^{14}-10^{16} \mathrm{~cm}^{-3}$ or, equivalently, less than $0.1 \%$ per ML, which act as $p$-type dopants $[38,39,41,42]$.

Based on this separation of isotropic (bulklike) and anisotropic surface contributions, we adopt the procedure applied to the $\mathrm{In} / \mathrm{Si}(111)$ system [38]. In this study, the change of the conductivity as a function of the oxygen dose $D$ could be well described via $\sigma_{\|}(D)=\sigma_{\mathrm{SCL}}+\sigma_{\|}(0) \exp -D / D_{0}[38]$. In this approach, the transport through the bulk is covered by $\sigma_{\mathrm{SCL}}$, which is almost constant during the dosage with molecular oxygen. In contrast, $\sigma_{\|}(0)$ denotes the surface transport along the wires. Thereby, both adsorption-induced changes of the band structure as well as changes of the lattice periodicity, giving rise to hopping transport, are subsumed by this contribution. Albeit this is a simple approach, it rather well describes our transport findings, i.e., that the $\mathrm{Si}(h h k)$ $\mathrm{Au}$ surfaces comprise differently reactive sites. In order to reveal further details regarding a band- or defect-mediated transport, temperature-dependent measurements would be necessary, which are out of the scope of this work. We will show below that, indeed, oxygen adsorption alters the band structure. In principle, defect-mediated transport across defects should be considered as well. However, the transport in the direction across the wires is rather independent to the adsorption, thus oxygen-induced hopping across the wires can be neglected. From Fig. 3(a) it is obvious that the impact of oxygen on $\mathrm{Si}(553)$-Au for the conductivity along the wires is quite small. Extrapolating the data with the above-mentioned approach yields an approximate value of $D_{0} \approx 200 \mathrm{~L}$. The same approach used for $\mathrm{Si}(557)$-Au [cf. with black dashed line in Fig. 3(b)] is only able to fit at best the behavior in the high dosage regime $(\geqslant 10 \mathrm{~L})$. We plotted also the fitting curve obtained for $\sigma_{\|,(553)}$ in Fig. 3(b) in order to show that the high dosage regime indeed reveals a similar dependence of conductance on oxygen exposure for both surfaces, possibly due to the reaction of oxygen at common structural motifs, e.g., the Si-HCs. Noticeably, the initial part of $\sigma_{\|,(557)}$ cannot be described by such an ansatz. We will show below that this deviating behavior is due to the presence of the $\mathrm{Si}$ adatoms on $\mathrm{Si}(557)-\mathrm{Au}$ and the oxygen reaction with them.

\section{DFT results}

In order to understand details of the oxygen adsorption process, DFT calculations were performed. Figures 4(a) and
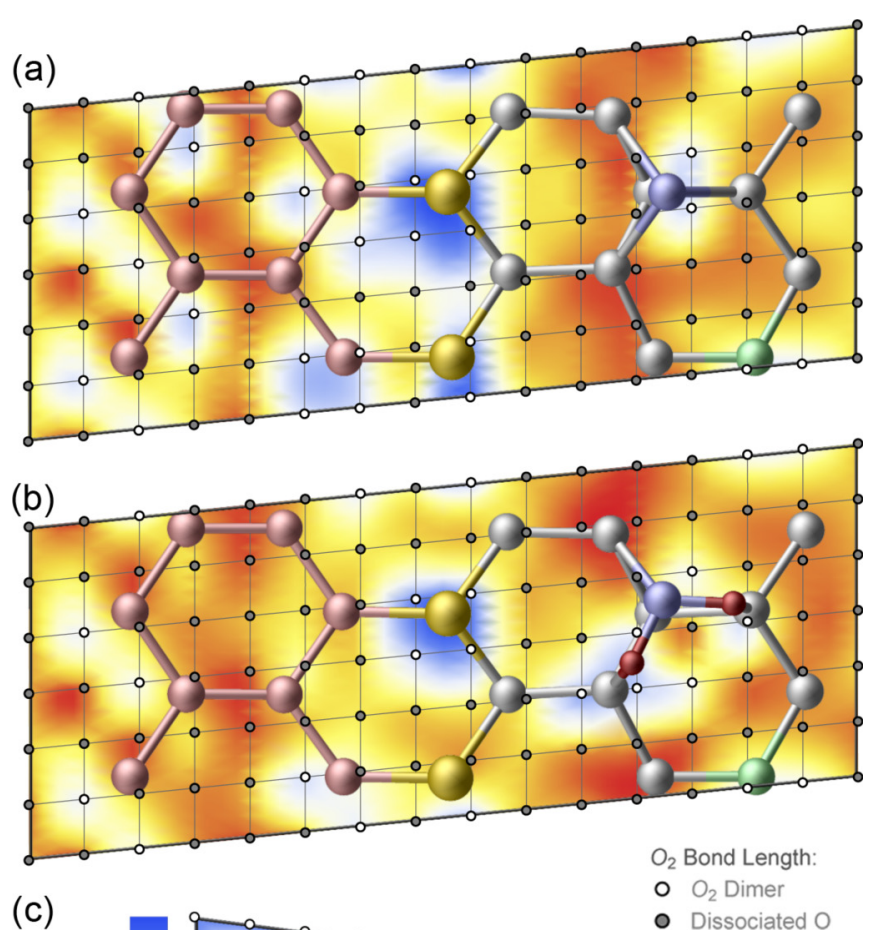

(c)

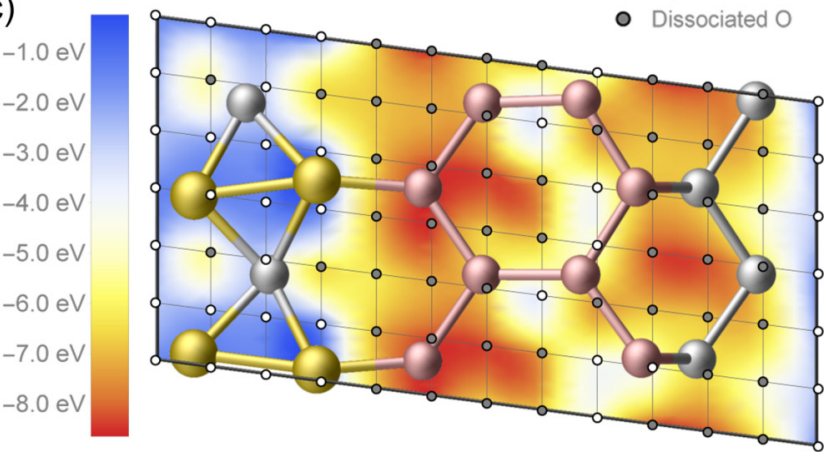

FIG. 4. Calculated adsorption energies for $\mathrm{O}_{2}$ adsorption within the unit cells of $\mathrm{Si}(557)-\mathrm{Au}$ (a) and $\mathrm{Si}(553)-\mathrm{Au}$ (c). The adsorption energies in (b) for $\mathrm{Si}(557)$-Au were determined with initial oxygen precoverage on the Si adatoms, indicated by red balls (see text). Tested adsorption sites of $\mathrm{O}_{2}$ are shown as small circles. Filled circles on the grid indicate where dissociative adsorption occurs spontaneously. The superimposed crystal structures refer to the structures of the clean (a), (c) and preoxidized surfaces (b) and illustrates the preferred adsorption sites. Au, O, Si-HC, and $\mathrm{Si}$ adatoms are marked by yellow, red, orange, and blue colors, respectively. The color coding of the energies is obtained by nonlinear bicubic interpolation.

4(c) show the adsorption energies for molecular oxygen adsorbed dissociatively on clean $\mathrm{Si}(557)-\mathrm{Au}$ and $\mathrm{Si}(553)-\mathrm{Au}$ at various lattice sites, respectively. In total, 90 and 72 different sites within the unit cells of $\mathrm{Si}(557)-\mathrm{Au}$ and $\mathrm{Si}(553)-\mathrm{Au}$ were probed.

It turns out that $\mathrm{O}$ adsorption at the Au sites is unfavorable for both surfaces. This is particularly pronounced in case of $\mathrm{Si}(553)$-Au. Oxygen clearly prefers to attack the Si-adatom and the Si-HC sites. This site-specific adsorption behavior for oxygen already explains the changes of the LEED patterns and disappearance of the $\times 2$ diffraction spots for $\mathrm{Si}(557)$ - $\mathrm{Au}$ upon adsorption, discussed in context with Fig. 1. Comparing the different $\mathrm{Si}$ adsorption sites on $\mathrm{Si}(557)-\mathrm{Au}$, the $\mathrm{Si}-\mathrm{HC}$ 

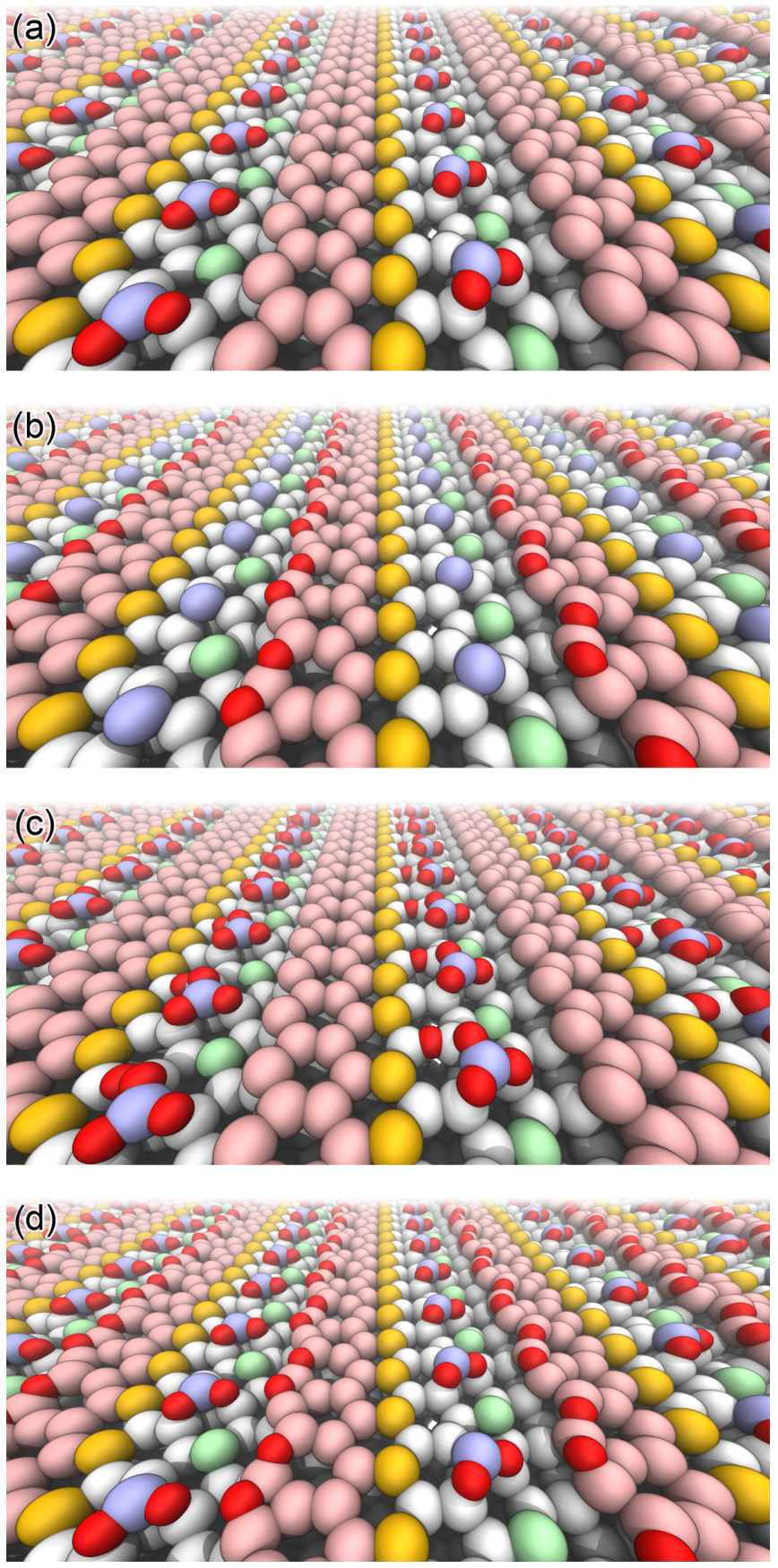

FIG. 5. Different models for the dissociative adsorption of molecular oxygen on $\mathrm{Si}(557)-\mathrm{Au}$. (a) Oxygen (dark red balls) bound to the Si-adatom (light blue) and rest-atom sites (light green). (b) Oxygen adsorption at the step edge of the $\mathrm{Si}-\mathrm{HC}$ structure (light red). (c) Further oxidation of the configuration shown in (a), where oxygen is now adsorbed next to Si rest atoms (green). (d) Alternative model for a twofold oxidation process, which is energetically less favorable by around $500 \mathrm{meV}$ compared to (c). However, in contrast to (c), both transport channels are affected by oxygen adsorption.

site is less favorable by around $250 \mathrm{meV}$ compared to $\mathrm{Si}$ adatoms. The corresponding adsorption geometries for these two preferred adsorption sites are shown in Figs. 5(a) and 5(b). The detailed analysis of seven different geometries in total showed that the configuration shown in Fig. 5(a) is energetically most favorable but nearly equivalent to a scenario, where the oxygen occupies slightly different adsorption sites around the $\mathrm{Si}$ adatoms. Concluding, a site-specific reaction of oxygen with the $\mathrm{Si}(557)-\mathrm{Au}$ surface at the $\mathrm{Si}$ adatoms is seen in DFT, but with randomly oriented $\mathrm{SiO}$ complexes destroying long-range order of the former $\mathrm{Si}$-adatom structure.

In the following, we will concentrate on the oxidation of $\mathrm{Si}(557)-\mathrm{Au}$. Inspired by a recent study of adsorption of molecular oxygen on $\mathrm{Si}(111)-7 \times 7$, we analyzed the possibility of subsequent oxidation processes [43]. Figure 4(b) shows the corresponding potential energy surface for a second step of oxidation (for guidance, the initial configuration of the preoxidized $\mathrm{Si}$-adatom sites is superimposed). Compared to the pristine $\mathrm{Si}(557)-\mathrm{Au}$ surface the barriers for further adsorption at the $\mathrm{Si}-\mathrm{HC}$ site, in proximity to the oxidized $\mathrm{Si}$ adatoms, are slightly increased. A likely configuration is shown in Fig. 5(c) and energetically favored by at least $500 \mathrm{meV}$ compared to configurations, where the two oxygen atoms of a molecule bind to different $\mathrm{Si}$ sites, e.g., to $\mathrm{Si}$ adatoms and Si-HCs, as shown in Fig. 5(d).

The corresponding band structures for $\mathrm{Si}(557)-\mathrm{Au}$ in comparison to $\mathrm{Si}(553)-\mathrm{Au}$ are shown in Fig. 6. Figures 6(a) and $6(\mathrm{~b})$ and $6(\mathrm{e})$ and $6(\mathrm{f})$ show the surface bands for the respective clean surfaces. As obvious, there are two bands crossing the Fermi energy $E_{F}$. Thereby, the green-colored band is induced by the Si rest atoms while the reddish-colored band stems mainly from the $\mathrm{Si}-\mathrm{HC}$ step-edge atoms. The $\mathrm{Si}$ adatoms give rise to the dispersing unoccupied band shown in blue. In Fig. 6(c), we show the Si(557)-Au band structure after oxidizing the Si adatoms and rest atoms [cf. Fig. 5(a)]. First of all, the former band of the Si adatoms (blue) shows strong admixtures of oxygen orbitals, coming along with localization. Second, the metallic band originating from the $\mathrm{Si}$ rest atoms is slightly shifted to higher energies and the electron effective mass at the Fermi energy increases by $13 \%$. Thus, the conductivity along the wire direction should be reduced. The electronegativity of the oxygen atoms obviously depopulates the bands associated with the rest atoms. Redistribution of charges associated with the oxidation process are the reason for formation of further surface dipoles which change the work function, as discussed in context of $\sigma_{\perp}$ for $\mathrm{Si}(557)-\mathrm{Au}$ [cf. Fig. 3(b)]. In contrast, the band originating from the Si-HC shows a weak anticrossing feature, but remains essentially unchanged. In agreement with the experiment, the DFT calculations revealed a reduction of the carriers and increase of their effective masses, leading to a reduced conductivity. However, the calculations were performed in the high coverage regime, where each of the Si-adatom and Si-rest-atom sites are covered by oxygen. Therefore, particularly in the low oxygen coverage regime, the mobility along the wires is further reduced by scattering at oxygen defects.

The band structure after the second step of oxidation depends crucially on details of the adsorption geometry. For instance, the bands for the energetically most favorable geometry, shown in Fig. 5(c), look rather similar to those obtained after the first oxidation step. In contrast, the scenario presented in Fig. 5(d) shows the strongest modifications in the band structure by opening an electronic gap of around $100 \mathrm{meV}$ at $E_{F}$. Of course, if the conductivity along the surface 
(a)
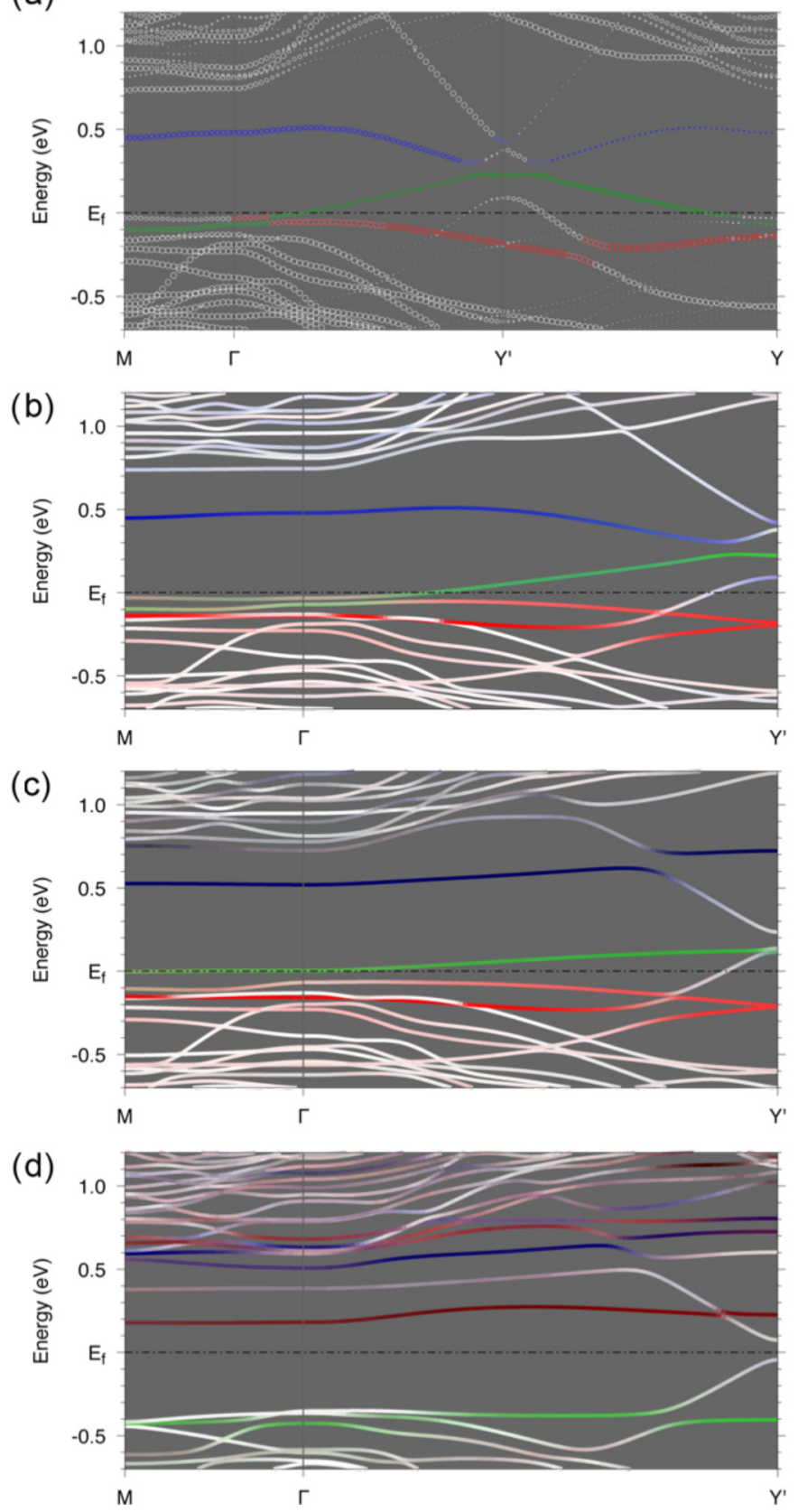

(e)
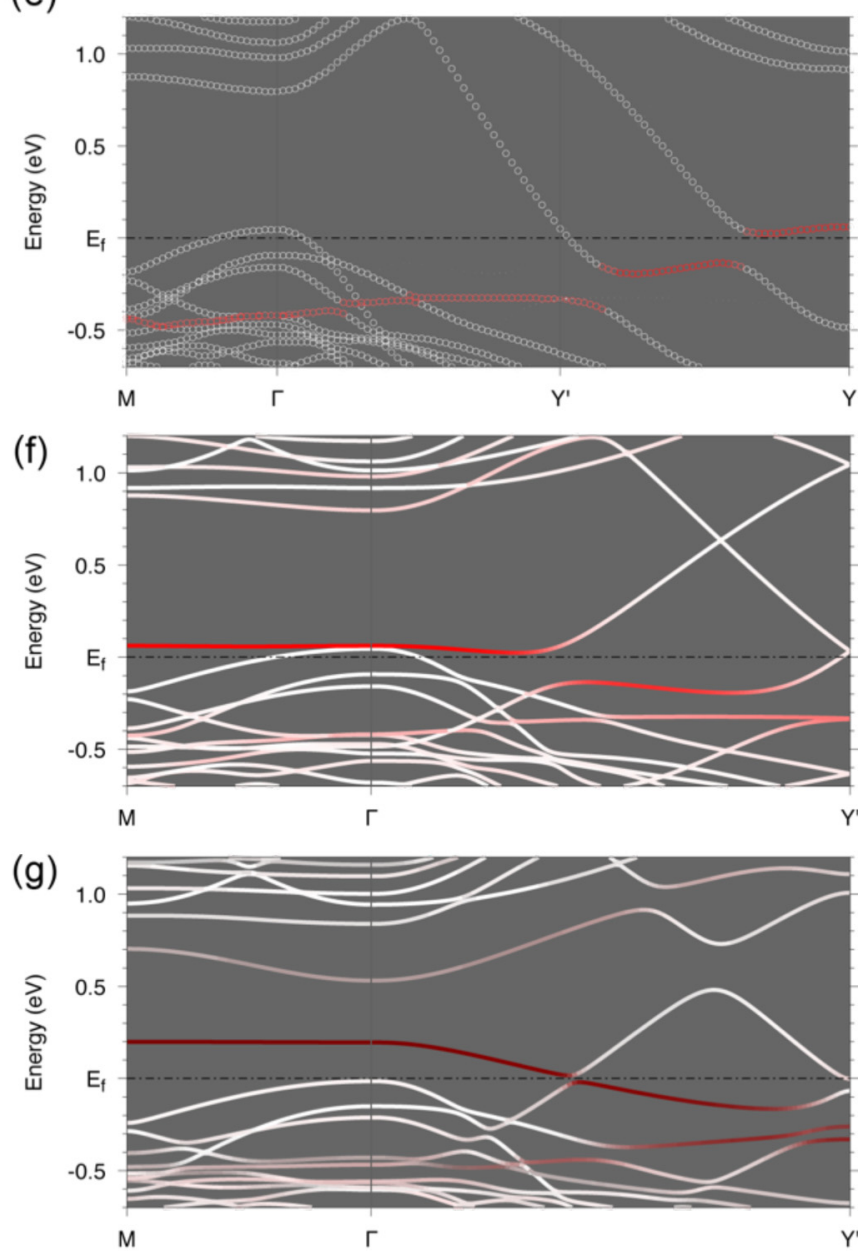

(h)

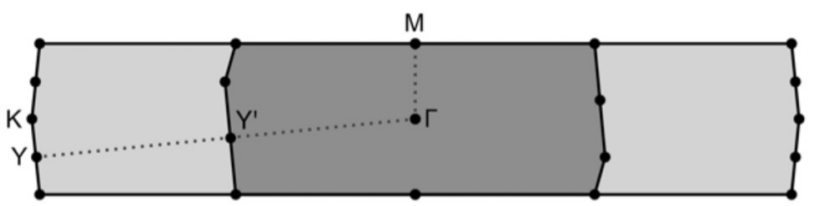

FIG. 6. Band structures for $\mathrm{Si}(557)-\mathrm{Au}$ and $\mathrm{Si}(553)-\mathrm{Au}$. Unfolded/folded $(\times 2)$ band structures for clean $\mathrm{Si}(557)-\mathrm{Au}$ and $\mathrm{Si}(553)-\mathrm{Au}$ surfaces are shown in (a), (b) and (e), (f), respectively. The point sizes in the unfolded bands structures (a) and (e) indicate the (5 $\times 1)$ character of the respective state (cf. [44]). Band structures for oxidation of Si(557) Si-adatom sites [cf. Fig. 5(a)] and Si-adatom and rest-atom sites [cf. Fig. 5(d)] are shown in (c) and (d), respectively. The band structure of $\mathrm{Si}(553)$-Au, where oxygen is built into the $\mathrm{Si}-\mathrm{HC}$ site, is shown in (g). The notation of the high-symmetry points is explained in (h). The colors of the surface bands denote their spatial localization: Si adatom (blue), Si rest atom (green), Si step-edge atoms (red). Dark blue (b), (c) denotes the admixture of oxygen orbitals upon adsorption.

band channels is entirely disabled, the anisotropy should vanish, similarly to the situation shown in Fig. 3(b). Albeit our theoretical calculations for the ideal surfaces revealed that the configuration shown in Fig. 5(d) is slightly less favorable than the geometry of Fig. 5(c), a reaction of these sites could be effectively triggered by surface defects. For instance, in case of water adsorption, STM revealed the occupation of Si-HC sites [22].
Obviously, the oxygen-modified electronic band structures discussed above describe only part of the influence of oxygen on the electron transport properties. For example, potentialwell scattering can be expected to play an important role as well [45]. Nonetheless, the DFT analysis showed that the $\mathrm{Si}(557)-\mathrm{Au}$ and $\mathrm{Si}(553)-\mathrm{Au}$ surfaces host differently reactive adsorption sites. Moreover, the $\mathrm{Si}(557)$-Au provides two reactive transport channels made up by $\mathrm{Si}$ atoms. For 
comparison, the metallic bands for the $\mathrm{Si}(553)-\mathrm{Au}$ surface stem mainly from the Au atoms [2]. The Si-HC band in this system is energetically slightly below $E_{F}$, similarly to the result obtained for $\mathrm{Si}(557)-\mathrm{Au}$, shown in Fig. 6(a). Therefore, oxygen adsorption at the $\mathrm{Au}$ sites is expected to yield the largest impact on transport in this system. However, since the $\mathrm{Au}$ strands are chemically inert, oxidation should have only a small influence on transport. In fact, the calculated band structures in Figs. 6(f) and 6(g) show that oxidation of the $\mathrm{Si}-\mathrm{HC}$ site at the $\mathrm{Si}(553)-\mathrm{Au}$ surface essentially shifts the $\mathrm{Si}$ step-edge states by about $0.1 \mathrm{eV}$, but has only a minor influence on the strongly dispersive Au states. Also in case of $\mathrm{Si}(557)-\mathrm{Au}$ it is found that the effect of the oxidation is stronger for the $\mathrm{Si}$ than for the Au states. Since the Si-adatom chain, that does not exist on $\mathrm{Si}(553)-\mathrm{Au}$, is also metallic on $\mathrm{Si}(557)-\mathrm{Au}$, the largest change in transport is due its oxidation.

\section{E. Quantitative analysis of the transport data}

We showed above that especially $\mathrm{Si}(557)-\mathrm{Au}$ is rather sensitive to adsorption of molecular oxygen. DFT revealed two transport channels, which originate primarily from different $\mathrm{Si}$-atom reconstructions. The reduction of the surface-state conductivity is mainly described by the change of the conductivity in each of the channels. Nonetheless, from an energetic point of view, theory showed that the oxidation of the $\mathrm{Si}$-adatom chain influences the adsorption energy of the $\mathrm{Si}-\mathrm{HC}$ site, which may give rise to a subsequent oxidation procedure. Although the energy differences are small compared to the adsorption energies, we will include this effect into the modeling of the transport data in the following, i.e., the relative coverages on each of the transport channels are not entirely independent.

In the following, $\Theta_{0}, \Theta_{1}$, and $\Theta_{2}$ denote the relative fractions of free, oxidized, and subsequent-oxidized sites on the $\mathrm{Si}(557)-\mathrm{Au}$ surface, respectively. Thereby, we assume that the subsequent-oxidation step occurs only if $\Theta_{1}$ is already nonzero. We are aware that this is a rather strict correlation, which may be less severe for the experiments performed at room temperature on surfaces with imperfections. Nonetheless, if the background pressure $p$ of oxygen (mass $m$ ) is kept constant during the experiment, the increase of subsequentoxidized sites per time is given by $\dot{\Theta}_{2}=C_{1} \Theta_{1}$. Accordingly, the rate for the increase of the singly oxidized states reads $\dot{\Theta}_{1}=C_{0} \Theta_{0}-\dot{\Theta}_{2}$. The $C_{i}$ 's are constants and comprise details about the sticking coefficients. Within the model of Langmuir adsorption, assuming first-order adsorption kinetics, i.e., no diffusion of the oxygen atoms after dissociation, the constants read as $C_{i}=c_{i} \exp \left(-E_{a}^{i} / k_{B} T\right) \sqrt{p / 2 \pi m k_{B} T}$, where the $c_{i}$ 's and the $E_{a}^{i}$, s denote the condensation coefficients and energy barriers for adsorption, respectively [46-48]. The combination of both rate equations leads to a second-order linear differential equation. Based on this subsequent oxidation model, the number of doubly oxidized sites follows $\Theta_{2}(t)=$ $\left[C_{0}-C_{0} e^{-C_{1} t}+C_{1}\left(e^{-C_{0} t}-1\right)\right] /\left(C_{0}-C_{1}\right)$. Finally, based on the above-mentioned equations, also $\Theta_{0}(t)$ and $\Theta_{1}(t)$ can be determined.

Assuming that during each oxidation step the initial resistivity $\rho_{0}$ is sectionally modified, the overall resistivity $\rho$ along the wires can be described via $\rho=\Theta_{0} \rho_{0}+\Theta_{1} \rho_{1}+\Theta_{2} \rho_{2}$,

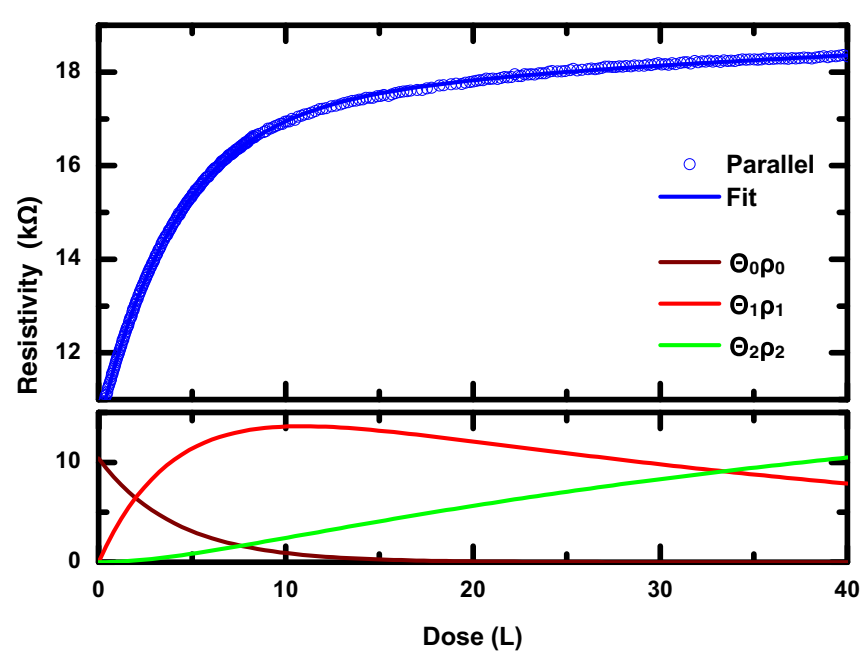

FIG. 7. Resistivity along the wires for $\mathrm{Si}(557)$-Au as a function of oxygen dose. The solid line is a fit to the data. The lower part shows the weighted contributions of the uncovered (brown curve, $\Theta_{0} \rho_{0}$ ), oxidized (red curve, $\Theta_{1} \rho_{1}$ ), and subsequent-oxidized fraction (green curve, $\left.\Theta_{2} \rho_{2}\right)$.

where the resistivity for the clean surface and the resistivities after the first $\left(\rho_{1}\right)$ and second step of oxidation $\left(\rho_{2}\right)$ are weighted by the number of available sites. Figure 7 shows the change of the resistivity as function of the oxygen dose. In addition, also the individual components are plotted. While the contribution $\Theta_{0} \rho_{0}$ gradually decreases, the other two oxidized states mutually increase. Thereby, the first oxidation dominates the initial increase of the resistivity, while the high dose regime is dominated by the subsequent oxidation.

According to this model, the increase of the resistivity due to oxidation at beginning of the dosing experiment can be approximated by $\Theta_{1} \rho_{1} \approx 1-\exp \left(-C_{0} D\right)$ (time $t$ equivalent to dose $D$ if $p=$ const). The value of $D_{0} \equiv 1 / C_{0}=4.1 \mathrm{~L}^{-1}$ is in good agreement with the decay constant found independently in the LEED experiment for the $\times 2$ reflexes. The corresponding contribution to the resistivity for an independent oxidation in the high coverage regime was shown and discussed by the dashed line in context of Fig. 3(b).

\section{SUMMARY AND CONCLUSION}

In summary, we performed a detailed SPA-LEED, transport, and DFT study on $\mathrm{Si}(h h k)$-Au systems. In particular, we elaborated in detail the effect of oxygen adsorption. Most strikingly, the origin for the metallic surface bands along the wires are of different origin. While the $\mathrm{Si}(553)$-Au surface turned out to be rather inert against oxidation, the $\mathrm{Si}(557)$-Au surface revealed a strong decrease of the conductance, which comes along with the complete destruction of the Si-adatom ordering along the chains. The DFT calculations were performed in the limit of high oxygen coverage and showed the reduction of charge carrier density and an increase of the effective masses and unambiguously confirmed the different orbital characters and reactivity of the transport channels. Any additional effects, e.g., due to disorder, were not covered by the calculations. Moreover, we were able to resolve a subsequent oxidation for $\mathrm{Si}(557)-\mathrm{Au}$ by high-resolution transport measurements. 
However, the surface transport comes along with a strong isotropic bulk contribution, which depends on the preparation and limits the resolution of our technique. High-resolution STM experiments should be performed in future in order to support further the structural models for adsorption and to elucidate in more detail any subsequent oxidation steps on these heterogeneous surfaces. Nonetheless, our investigation showed that the 1D transport channels, realized by $\mathrm{Si}(h h k)$ surfaces with quite similar structural elements, have different

[1] C. Zeng, P. R. C. Kent, T.-H. Kim, A.-P. Li, and H. H. Weitering, Nat. Mater. 7, 539 (2008).

[2] S. C. Erwin and F. Himpsel, Nat. Commun. 1, 58 (2010).

[3] B. Hafke, T. Frigge, T. Witte, B. Krenzer, J. Aulbach, J. Schäfer, R. Claessen, S. C. Erwin, and M. Horn-von Hoegen, Phys. Rev. B 94, 161403(R) (2016).

[4] H. Okino, R. Hobara, I. Matsuda, T. Kanagawa, S. Hasegawa, J. Okabayashi, S. Toyoda, M. Oshima, and K. Ono, Phys. Rev. B 70, 113404 (2004).

[5] H. Okino, I. Matsuda, R. Hobara, S. Hasegawa, Y. Kim, and G. Lee, Phys. Rev. B 76, 195418 (2007).

[6] H. Okino, I. Matsuda, S. Yamazaki, R. Hobara, and S. Hasegawa, Phys. Rev. B 76, 035424 (2007).

[7] P. C. Snijders, S. Rogge, and H. H. Weitering, Phys. Rev. Lett. 96, 076801 (2006).

[8] J. N. Crain and D. T. Pierce, Science 307, 703 (2005).

[9] W. G. Schmidt, M. Babilon, C. Thierfelder, S. Sanna, and S. Wippermann, Phys. Rev. B 84, 115416 (2011).

[10] I. Song, D.-H. Oh, H.-C. Shin, S.-J. Ahn, Y. Moon, S.-H. Woo, H. J. Choi, C.-Y. Park, and J. R. Ahn, Nano Lett. 15, 281 (2015).

[11] M. Krawiec and M. Jałochowski, Phys. Rev. B 82, 195443 (2010).

[12] G. Lee, S.-Y. Yu, H. Shim, W. Lee, and J.-Y. Koo, Phys. Rev. B 80, 075411 (2009).

[13] T. Shibasaki, N. Nagamura, T. Hirahara, H. Okino, S. Yamazaki, W. Lee, H. Shim, R. Hobara, I. Matsuda, G. Lee, and S. Hasegawa, Phys. Rev. B 81, 035314 (2010).

[14] S.-W. Kim and J.-H. Cho, Phys. Rev. B 93, 241408(R) (2016).

[15] H. W. Yeom, D. M. Oh, S. Wippermann, and W. G. Schmidt, ACS Nano 10, 810 (2016).

[16] S. C. Erwin, I. Barke, and F. J. Himpsel, Phys. Rev. B 80, 155409 (2009).

[17] A. Stępniak, M. Krawiec, G. Zawadzki, and M. Jałochowski, J. Phys.: Condens. Matter 24, 095002 (2012).

[18] W. H. Choi, P. G. Kang, K. D. Ryang, and H. W. Yeom, Phys. Rev. Lett. 100, 126801 (2008).

[19] J. N. Crain, J. L. McChesney, F. Zheng, M. C. Gallagher, P. C. Snijders, M. Bissen, C. Gundelach, S. C. Erwin, and F. J. Himpsel, Phys. Rev. B 69, 125401 (2004).

[20] I. Barke, S. Polei, V. v. Oeynhausen, and K.-H. Meiwes-Broer, Phys. Rev. Lett. 109, 066801 (2012).

[21] K.-D. Ryang, P. G. Kang, H. W. Yeom, and S. Jeong, Phys. Rev. B 76, 205325 (2007).

[22] P.-G. Kang, J. S. Shin, and H. W. Yeom, Surf. Sci. 603, 2588 (2009).

[23] M. Krawiec, Phys. Rev. B 81, 115436 (2010).

[24] J. P. Ibe, P. P. Bey, Jr., S. L. Brandow, R. A. Brizzolara, N. A. Burnham, D. P. DiLella, K. P. Lee, C. R. K. Marrian, and R. J. Colton, J. Vac. Sci. Technol. A 8, 3570 (1990). origins. Consequently, it is difficult to generalize the impact of adsorbates.

\section{ACKNOWLEDGMENTS}

Financial support by the Deutsche Forschungsgemeinschaft (DFG) through the Research Unit FOR1700 (projects E4 and T1) is gratefully acknowledged.

[25] J. P. Perdew, K. Burke, and M. Ernzerhof, Phys. Rev. Lett. 77, 3865 (1996).

[26] P. E. Blöchl, Phys. Rev. B 50, 17953 (1994).

[27] G. Kresse and J. Furthmueller, Phys. Rev. B 54, 11169 (1996).

[28] S. C. Erwin and P. C. Snijders, Phys. Rev. B 87, 235316 (2013).

[29] T. Nagao, S. Yaginuma, T. Inaoka, and T. Sakurai, Phys. Rev. Lett. 97, 116802 (2006).

[30] I. Song, J. S. Goh, S.-H. Lee, S. W. Jung, J. S. Shin, H. Yamane, N. Kosugi, and H. W. Yeom, ACS Nano 9, 10621 (2015).

[31] T. Lichtenstein, C. Tegenkamp, and H. Pfnür, J. Phys.: Condens. Matter 28, 354001 (2016).

[32] T. Lichtenstein, J. Aulbach, J. Schäfer, R. Claessen, C. Tegenkamp, and H. Pfnür, Phys. Rev. B 93, 161408(R) (2016).

[33] D. Sánchez-Portal, S. Riikonen, and R. M. Martin, Phys. Rev. Lett. 93, 146803 (2004).

[34] J. A. Lipton-Duffin, J. M. MacLeod, and A. B. McLean, Phys. Rev. B 73, 245418 (2006).

[35] J. R. Ahn, P. G. Kang, K. D. Ryang, and H. W. Yeom, Phys. Rev. Lett. 95, 196402 (2005).

[36] I. Miccoli, F. Edler, H. Pfnür, and C. Tegenkamp, J. Phys.: Condens. Matter 27, 223201 (2015).

[37] T. Kanagawa, R. Hobara, I. Matsuda, T. Tanikawa, A. Natori, and S. Hasegawa, Phys. Rev. Lett. 91, 036805 (2003).

[38] F. Edler, I. Miccoli, S. Demuth, H. Pfnür, S. Wippermann, A. Lücke, W. G. Schmidt, and C. Tegenkamp, Phys. Rev. B 92, 085426 (2015).

[39] T. Uetake, T. Hirahara, Y. Ueda, N. Nagamura, R. Hobara, and S. Hasegawa, Phys. Rev. B 86, 035325 (2012).

[40] T. Tanikawa, I. Matsuda, T. Kanagawa, and S. Hasegawa, Phys. Rev. Lett. 93, 016801 (2004).

[41] T. Hirahara, I. Matsuda, C. Liu, R. Hobara, S. Yoshimoto, and S. Hasegawa, Phys. Rev. B 73, 235332 (2006).

[42] H. M. Zhang, K. Sakamoto, G. V. Hansson, and R. I. G. Uhrberg, Phys. Rev. B 78, 035318 (2008).

[43] C.-Y. Niu and J.-T. Wang, J. Chem. Phys. 139, 194709 (2013).

[44] P. V. C. Medeiros, S. Stafström, and J. Björk, Phys. Rev. B 89, 041407(R) (2014).

[45] S. Wippermann, N. Koch, and W. G. Schmidt, Phys. Rev. Lett. 100, 106802 (2008).

[46] I. Langmuir, J. Am. Chem. Soc. 40, 1361 (1918).

[47] H. Lüth, Solid Surfaces, Interfaces and Thin Films, Graduate Texts in Physics (Springer, Berlin, 2010), pp. 517-559.

[48] K. W. Kolasinski and K. K. Kolasinski, Surface Science: Foundations of Catalysis and Nanoscience (Wiley, Hoboken, NJ, 2012). 